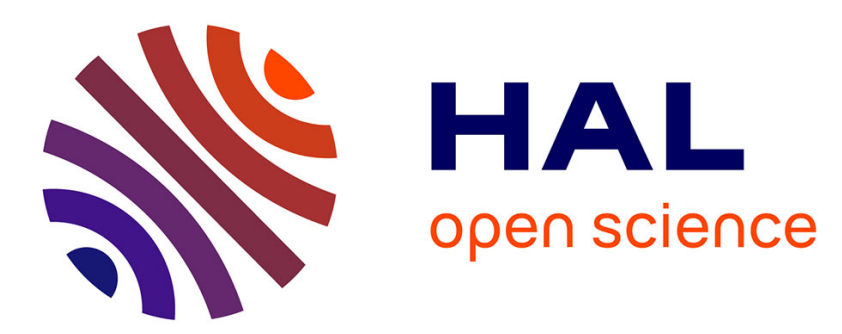

\title{
Genetic variation of loci potentially under selection confounds species-genetic diversity correlations in a fragmented habitat
}

\author{
Angeline Bertin, Nicolas Gouin, Alex Baumel, Ernesto Gianoli, Juan \\ Serratosa, Rodomiro Osorio, Stephanie Manel
}

\section{To cite this version:}

Angeline Bertin, Nicolas Gouin, Alex Baumel, Ernesto Gianoli, Juan Serratosa, et al.. Genetic variation of loci potentially under selection confounds species-genetic diversity correlations in a fragmented habitat. Molecular Ecology, 2017, 26 (2), pp.431 - 443. 10.1111/mec.13923 . hal-01605033

\section{HAL Id: hal-01605033 https://hal.science/hal-01605033}

Submitted on 25 Apr 2018

HAL is a multi-disciplinary open access archive for the deposit and dissemination of scientific research documents, whether they are published or not. The documents may come from teaching and research institutions in France or abroad, or from public or private research centers.
L'archive ouverte pluridisciplinaire HAL, est destinée au dépôt et à la diffusion de documents scientifiques de niveau recherche, publiés ou non, émanant des établissements d'enseignement et de recherche français ou étrangers, des laboratoires publics ou privés. 


\title{
Genetic variation of loci potentially under selection confounds species-genetic diversity correlations in a fragmented habitat
}

\author{
ANGELINE BERTIN ${ }^{1}$, NICOLAS GOUIN ${ }^{1,2,3}$, ALEX BAUMEL ${ }^{4}$, ERNESTO \\ GIANOLI $^{1,5}$, JUAN SERRATOSA ${ }^{6}$, RODOMIRO OSORIO ${ }^{1}$ and STEPHANIE MANEL $^{7}$ \\ ${ }^{1}$ Departamento de Biología, Universidad de La Serena, La Serena, Chile \\ ${ }^{2}$ Centro de Estudios Avanzados en Zonas Áridas, La Serena, Chile \\ ${ }^{3}$ Instituto de Investigación Multidisciplinar en Ciencia y Tecnología, Universidad de La \\ Serena, La Serena, Chile \\ ${ }^{4}$ Institut Méditerranéen de Biodiversité et d'Ecologie marine et continentale (IMBE) \\ Aix Marseille Univ, Univ Avignon, CNRS, IRD, Marseille, France. \\ ${ }^{5}$ Departamento de Botánica, Universidad de Concepción, Concepción, Chile \\ ${ }^{6}$ Facultad de Ciencias del Mar, Doctorado en Biología y Ecología Aplicada, Universidad \\ Católica del Norte \\ ${ }^{7}$ EPHE, PSL Research University, CNRS, UM, SupAgro, IRD, INRA, UMR 5175 CEFE, \\ F-34293 Montpellier, FRANCE
}

Keywords: community genetics, species-genetic diversity correlations, outlier loci, habitat connectivity, high Andean wetlands

\section{Corresponding Author:}

Angéline Bertin

Departamento de Biología, Facultad de Ciencias, Universidad de La Serena, Av. Raúl Bitrán 1305, Casilla 554, La Serena, Chile.

Fax : (56) (51) 2 204383, electronic mail address: abertin@userena.cl;

Running Title: Influence of outlier loci on SGDCs 


\section{Abstract}

2 Positive species-genetic diversity correlations (SGDCs) are often thought to result from the

3 parallel influence of neutral processes on genetic and species diversity. Yet, confounding effects

4 of non-neutral mechanisms have not been explored. Here, we investigate the impact of non-

5 neutral genetic diversity on SGDCs in high Andean wetlands. We compare correlations between

6 plant species diversity (SD) and genetic diversity (GD) calculated with and without loci

7 potentially under selection (outlier loci). The study system includes 2188 specimens from five

8 species (three common aquatic macroinvertebrate and two dominant plant species) that were

9 genotyped for 396 amplified fragment length polymorphism loci. We also appraise the

10 importance of neutral processes on SGDCs by investigating the influence of habitat

11 fragmentation features. Significant positive SGDCs were detected for all five species (mean

12 SGDC $=0.52 \pm 0.05$ ). While only a few outlier loci were detected in each species, they resulted

13 in significant decreases in GD and in SGDCs. This supports the hypothesis that neutral processes

14 drive species-genetic diversity relationships in high Andean wetlands. Unexpectedly, the effects

15 on genetic diversity of the habitat fragmentation characteristics in this study increased with the

16 presence of outlier loci in two species. Overall, our results reveal pitfalls in using habitat features

17 to infer processes driving SGDCs and show that a few loci potentially under selection are enough

18 to cause a significant downward bias in SGDC. Investigating confounding effects of outlier loci

19 thus represents a useful approach to evidence the contribution of neutral processes on species-

20 genetic diversity relationships. 


\section{Introduction}

22 Investigating spatial or temporal patterns of covariation between species diversity within a

23 community (SD) and genetic diversity within a species (GD) is one of the main objectives

24 of community genetics (Antonovics 1992). It is a key step towards a conceptual synthesis at

25 the interface between ecology and evolutionary biology (Vellend \& Geber 2005). It is also

26 a core issue in conservation biology as it provides valuable information to understand and

27 predict different facets of biodiversity (Hu et al. 2009; Lavergne et al. 2010; Mouquet et al.

28 2012).

29 Studies addressing species genetic diversity correlations (SGDCs) in the wild have

30 shown that the relationship between GD and SD can take different forms depending on the

31 mechanisms at work (Kahilainen et al. 2014), with reports of positive (He et al. 2008;

32 Papadopoulou et al. 2011; Lamy et al. 2013), negative (Silvertown et al. 2009; Xu et al.

33 2016) and no correlation (Taberlet et al. 2012) between the two components. Concordant

34 spatial patterns between species and genetic diversity can emerge from various processes.

35 They may result from direct causal relationships between the two biodiversity components

36 (Vellend and Geber 2005). For instance, GD can influence SD when genetic variation of

37 habitat-forming species, such as forest trees, structures dependent communities (i.e. micro-

38 organisms, fungi, arthropods and vertebrates, Whitham et al. 2006). Alternatively, SD in

39 the community can impose variable selective pressures on populations, and therefore alter

40 the levels of population GD (Strauss et al. 2005; Vellend and Geber 2005; Johnson and

41 Stinchcombe 2007; Lankau and Strauss 2007). SD and GD can become indirectly

42 associated as well, by responding similarly to common mechanisms (Vellend and Geber

43 2005). Indeed, both ecological and genetic models posit similar effects of drift and 
44 migration on species and genetic diversity (e.g. depletion effects of drift and diversity

45 increases from immigration, Hu et al. 2009). Thus, concordant spatial patterns between

46 species and genetic diversity can be common under conditions of demographic stochasticity

47 and dispersal limitation (Rosenzweig 1995; Frankham 1996; Frankham 1997). To date,

48 little research has been conducted into the drivers of negative SGDCs (Xu et al. 2016).

49 According to a modeling study, negative SGDCs can occur under particular conditions of

50 high genetic mutation rates, depending on the relative importance of mutation, immigration

51 and local competition (Laroche et al. 2015). They may also occur in situations where

52 increases in SD, promoted by environmental heterogeneity, are associated with a reduction

53 in niche breadth and/or population size (Taberlet et al. 2012; Vellend et al. 2014; Xu et al.

54 2016).

55 While both neutral and selective mechanisms can generate positive covariation

56 between genetic and species diversity (Vellend and Geber 2005), evidence suggests that

57 neutral processes play a dominant role of in this respect (Odat et al. 2004; Vellend 2004;

58 Struebig et al. 2011; Papadopoulou et al. 2011; Lamy et al. 2013; Vellend et al. 2014).

59 Positive SGDCs are common in discrete habitat units like oceanic islands, lakes, or forest

60 fragments, and less frequent in arbitrarily defined spatial units such as grid cells or

61 vegetation plots (Vellend and Geber 2005; Whitlock 2014; Vellend et al. 2014). In

62 fragmented landscapes, migration and drift are likely major contributors to within-patch

63 diversity. Thus, positive SGDCs among islands and habitat patches have been postulated to

64 arise from the parallel influence of neutral processes on both species and genetic diversity

65 (Vellend and Geber 2005; Whitlock 2014; Vellend et al. 2014). This is supported by a

66 number of empirical studies, which have demonstrated that local habitat characteristics

67 influencing rates of stochastic immigration and extinction contribute markedly to species- 
genetic diversity relationships in fragmented habitats (Vellend 2005; He et al. 2008;

69 Papadopoulou et al. 2011; Lamy et al. 2013). These habitat features include habitat size, connectivity and perturbation. Indeed, both habitat carrying capacity and heterogeneity are

71 expected to increase with habitat size. Larger patches can thus support larger populations,

72 which are both more genetically diverse and more resilient to genetic drift (Frankham

73 1997). In addition, by offering more diverse ecological niches, larger habitats are also

74 expected to hold more diverse communities, and are consequently less susceptible to

75 random species loss through ecological drift (MacArthur and Wilson 1967, Hubbell 2001).

76 Because habitat connectivity influences rates of migration, it is expected to result in

77 increases in both genetic and species diversity, by facilitating the introduction of new

78 alleles in a population or new species in the community (Hu et al. 2009). Regarding habitat

79 perturbation, it can cause parallel effects on species and genetic diversity by provoking

80 extinction of species and alleles irrespectively of their identities (Vellend 2003).

81 Neutral explanations for positive SGDCs have also come from the wide use of

82 presumed neutral molecular markers to estimate genetic diversity in SGDC investigations

83 (Vellend and Geber 2005; Vellend et al. 2014). Genetic variation of molecular markers

84 with supposedly no effects on fitness, such as microsatellite or amplified fragment length

85 polymorphism (AFLP) loci, is considered to mirror neutral processes, including mutation,

86 genetic drift and gene flow (Holderegger et al. 2006; Kirk and Freeland 2011). SGDCs

87 estimated with these markers have therefore been assumed to emerge from concordant

88 changes in species and genetic diversity induced by neutral processes (Vellend and Geber

89 2005; Lamy et al. 2013; Vellend et al. 2014). However, no attention has been paid to

90 confounding effects of non-neutral mechanisms, which may lead to erroneous conclusions

91 regarding the mechanisms causing SD-GD covariation (Fig. 1). Indeed, selection generates 
92 changes in allele frequencies at target genes, but also in adjacent, linked regions (genetic

93 hitchhiking; Barton 2000). Such linked DNA regions, even non-coding DNA, can thus bear

94 signatures of selection (confounding effect 1 in Fig. 1; Barton 2000; Oleksyk et al. 2010).

95 When used to infer neutral processes, such loci may bias population parameter estimates

96 (Luikart et al. 2003). Their expected effects on genetic diversity depend on the type of

97 selection operating, as each selection mode leads to specific patterns of allele frequency

98 changes (Oleksyk et al. 2010). For instance, divergent directional selection decreases

99 genetic variation within populations, while balancing selection maintains variation within

100 populations. A few loci potentially under divergent selection are enough to cause a

101 significant downward bias in the assessment of GD estimated from markers assumed to be

102 neutral (García-Verdugo et al. 2015). The presence of such markers can thus be misleading

103 for SGDC studies. When SGDCs are driven by neutral mechanisms, loci under selection

104 are likely to decrease the correlation between species and genetic diversity, since only

105 neutral GD is expected to covary with SD in such circumstances. In contrast, no such

106 effects are expected when SGDCs arise from non-neutral processes.

107 Separating the neutral and non-neutral components of GD not only opens new

108 perspectives for the study of SGDCs, but can also be critical to interpreting apparent effects

109 of local habitat features in a neutral framework. Several studies have investigated how

110 disturbance regime, habitat size and/or connectivity relate to SGDCs in order to infer

111 underlying mechanisms (Vellend and Geber 2005; Papadopoulou et al. 2011; Lamy et al.

112 2013; Vellend et al. 2014). These studies postulate that these local features become

113 associated with neutral components of SD/GD due to their modulating role on rates of

114 migration and stochastic loss of species/alleles. However, these relationships can be

115 mistakenly attributed to neutral processes (confounding effect 2 in Fig. 1) if disturbance 
116 regime, habitat size and/or connectivity and selective environmental factors are spatially

117 correlated, as they would lead to indirect associations between non-neutral components of

118 GD and the local habitat features. These confounding effects have been documented for

119 species richness-island area associations (Ricklefs and Lovette 1999), and are expected at

120 the genetic level since populations at the margins of a species distribution range are not

121 only more fragmented and isolated than central populations, but also subject to different

122 ecological constraints (central-marginal hypothesis, Eckert et al. 2008). In such situations,

123 factoring out the effects of disturbance regime, habitat size and/or connectivity bears the

124 risk of overestimating the contribution of neutral processes on SD and GD, as it is likely to

125 control for some influence of selective factors as well.

126 In this study, we aimed to disentangle the confounding effects of non-neutral

127 genetic diversity on species-genetic diversity relationships in a highly fragmented

128 ecosystem, the high Andean wetlands of Chile's Norte Chico. These wetlands, formed by

129 groundwater upwelling, resemble oases in an arid matrix (Fig. 2, Squeo et al. 2006). They

130 occupy a latitudinal gradient of both aridity (increasing northwards) and wetland density

131 (Fig. 2). This suggests that wetlands differing in connectivity also experience different

132 selective pressures. Given the high degree of fragmentation of these ecosystems, we

133 hypothesized that neutral processes should play a critical role in species-genetic diversity

134 relationships. Thus, we expected loci influenced by selection to decrease SGDCs. We also

135 postulated that such loci can complicate the evaluation of the importance of neutral

136 processes on SGDC by factoring out the effects of habitat connectivity on SD and GD.

137 Indeed, due to the spatial covariation between habitat fragmentation and the latitudinal

138 environment gradient, GD of loci under environmental gradient pressure is also expected to

139 correlate with fragmentation characteristics. To test these conjectures, we investigated 
140 SGDCs between species diversity of high Andean wetland vegetation and GDs of five

141 species (two plants and three macroinvertebrates) with contrasting life-histories. We used a

142 genome scan approach to screen for AFLP markers potentially under selection (i.e. outlier

143 loci; Storz et al. 2004) and analyzed SGDCs and the effects of habitat characteristics (size,

144 connectivity and stability) on SGDCs with and without these loci. Our results showed that,

145 as expected, confounding effects of outlier loci on GD decrease SGDCs. This trend was

146 particularly evident with loci potentially under divergent selection. We also found that, in

147 some species, GD at outlier loci correlate with habitat features supposedly linked to neutral

148 processes, revealing pitfalls in using such characteristics to infer processes driving SGDCs.

$150 \quad$ Material and methods

151 Study system

152 We analyzed species and genetic diversity for 21 high Andean wetlands located along a ca.

$153600 \mathrm{~km}$ latitudinal range $\left(26^{\circ} \mathrm{S}-32^{\circ} \mathrm{S}\right)$ in north-central Chile (Fig. 2, and see Bertin et al.

154 2015). The region, known as Norte Chico, is a biodiversity hotspot, and is characterized by

155 remarkably high levels of endemism (Arroyo et al. 2004). It includes five hydrologically

156 unconnected river basins. The climate of the region varies from hyperarid in the north to

157 mediterranean in the south, with mean annual precipitation ranging between $35 \mathrm{~mm}$ and

$158200 \mathrm{~mm}$ for the northernmost and southernmost valleys, respectively.

159

160 Data collection

161 Community and population sampling 
162 Sampling of plant and benthic macroinvertebrate specimens was carried out between March

163 and April 2011. For plant diversity assessment, the length of each wetland was divided into

164 five sectors and a $30 \times 30 \mathrm{~cm}$ quadrat was randomly placed within each sector. Plant

165 species were separated and identified in the laboratory.

166 For genetic diversity evaluation, we selected five common species: two dominant

167 plants, Carex gayana (Cyperaceae) and Patosia clandestina (Juncaceae), and three

168 abundant aquatic macroinvertebrates, Andesiops peruvianus (Insecta, Ephemeroptera),

169 Austrelmis sp. (Insecta, Coleoptera) and Hyallela fossamancinii (Crustacea, Amphipoda).

170 Samples were collected by hand or using a small fishing net in the case of aquatic

171 organisms. The entire wetland surface area was sampled in each case, with individual

172 wetlands ranging in size from 3.7 to 38 ha. Leaf samples were conserved individually in

173 silica gel until DNA extraction, whereas macroinvertebrates were stored in 95\% alcohol.

174 The plant C. gayana was the only species found to occur within all 21 wetlands sampled.

175 Details regarding sampling sites and size for each species are given in Table S1.

177 Genetic data

178 For all five species, DNA extraction was performed using a CTAB protocol. AFLP

179 amplifications and genotyping were carried out using standard procedures and following

180 the protocol of Meudt and Clarke (2007), available at http://clarkeresearch.org/aflp_2012-

181 01-26/aflp.html, with very few modifications. In each species, four combinations of

182 specific primers were selected based on their reliability and number of fragments amplified.

183 AFLP fragments were separated by capillary electrophoresis and genotyping was

184 performed with the software GeneMarker v2.4 (Softgenetics). Following Bonin et al.

185 (2007), we retained only AFLP loci with genotyping error rate $<10 \%$, with the exception 
186 of the crustacean $H$. fossamancinii, for which we included loci with up to $12 \%$ genotyping

187 error in order to maintain a comparable number of loci for all species. Loci with redundant

188 information were filtered using the software AFLPOP (Duchesne and Bernatchez 2002).

189 Detailed procedures and AFLP primer pairs are described in Supplementary Information.

190 Only polymorphic markers (those with band frequencies between 5\% and 95\%) were kept

191 in the analysis (Bonin et al. 2007).

193 Habitat parameters

194 Habitat attributes associated with connectivity, size and stability were assessed for each of 195 the 21 sampled wetlands. Attributes were derived from maps of wetland distribution

196 generated based on spectral analysis of Landsat 8 OLI satellite imagery

197 (http://glovis.usgs.gov/, see Fig. 2) and elevation data. Normalized Difference Vegetation

198 Index, a measure of actively photosynthesizing vegetation (NDVI, Rouse et al. 1973), was

199 first calculated using Landsat data (January 2011) for a training dataset of 87 high Andean

200 wetlands mapped on-site in the Norte Chico region by Chile's Ministry of Environment and

201 Agricultural and Livestock Service. Based on the mean NDVI value of all pixels

202 comprising the 87 field-mapped wetlands, and applying the minimum observed elevation

203 value as a cut-off, we defined wetlands here as all vegetated pixels with NDVI values $>0.2$,

204 located above $1800 \mathrm{~m}$ a.s.l. To exclude sparse vegetation from the mapping, only vegetated

205 areas $\geq 0.36$ ha were considered.

206 We calculated various local structural connectivity metrics for each wetland using

207 Graphab 1.0 (Foltete et al. 2012), including node degree (Dg), clustering coefficient (CC),

208 closeness centrality (CCe), eccentricity (Ec), betweenness centrality (BC) and wetland

209 density. These metrics were quantified by considering all the wetlands mapped within a 20 
$210 \mathrm{~km}$ radius from the focal site. In addition, we estimated the surface of each focal wetland

211 from the GIS-based map (Fig. 2). As a proxy for wetland stability, we used the temporal

212 range of the mean NDVI of each site calculated over 20 years (from 1991 to 2002 and from

2132004 to 2011). Before evaluating the impact of the habitat metrics on diversity, we removed

214 strongly correlated predictors by excluding one variable when a Pearson's correlation

215 between a pair of habitat metrics was $>0.7$. The final set of predictors included wetland

216 size, Dg, CCe, Ec, BC and the temporal range of NDVI wetland values.

\section{Statistical analysis}

\section{Identification of outlier loci}

220 We used the Bayesian likelihood $F_{\text {ST }}$-based method implemented in the software BayeScan

221 (Foll and Gaggiotti 2008) to identify outlier loci. This approach estimates for each locus the

222 posterior probabilities of two alternative models: a neutral one and a model including

223 selection. We performed the analyses using the default parameters and considered a

224 conservative value of 10 for the prior odds (10:1 odds in favor of the neutral model). Loci

225 with $F_{\text {ST }}$ values substantially higher than the observed norm are considered potentially

226 under diversifying selection, while those with substantially lower $F_{\text {ST }}$ values are considered

227 potentially under balancing or purifying selection (Foll and Gaggiotti 2008). These outlier

228 loci were identified with the R function plot_bayescan as described in the user's manual,

229 using a false discovery rate (FDR, the q-value threshold) of 5\%. Accordingly, we examined

230 the effects of outlier loci by creating three marker datasets and considering their $F_{\text {ST }}$ values:

231 a dataset free of outliers (DS1), one with all the genotyped loci, thus including both the 
232 non-outlier and all the outlier loci (i.e. with high and low $F_{\mathrm{ST}}$, DS2) and one dataset

233 including the non-outlier loci and the outlier loci with high $F_{\text {ST }}$ only (DS3).

Species richness, genetic diversity and the influence of outlier loci on genetic diversity estimates

237 We used the statistical software R (R Core Team 2015) to calculate diversity indices and 238 perform SGDC analyses. We estimated species diversity within each wetland as species

239 richness of all the collected quadrats, and within-population genetic diversity as Nei's gene

240 diversity (Nei 1987). We calculated the bootstrapped 95\% confidence interval of species

241 richness and Nei's indices by considering 10,000 bootstrap replications using the $\mathrm{R}$

242 package rich (Rossi 2011) and R functions of AFLPdat (Ehrich 2006), respectively. Nei's

243 gene indices were computed for the three marker datasets separately (DS1, DS2 and DS3).

244 We tested the effects of outlier loci on GD by carrying out linear mixed models (LMM) of

245 Nei's gene diversity (dependent variable) in relation to the presence of outlier loci (fixed

246 effect), and including species and site as crossed random factors. We performed two such

247 analyses, testing the effects of all the outlier loci (with datasets DS1 and DS2), and of those

248 with high $F_{\text {ST }}$ only (with datasets DS1 and DS3). These analyses were also performed for

249 each species separately.

\section{Effects of habitat characteristics on species and genetic diversity}

252 We used partial least-square (PLS) regressions to analyze the influence of habitat

253 characteristics on species and genetic diversity. PLS regressions are recommended in

254 analyses involving a large number of potentially correlated predictor variables and few

255 observations (Mevik and Wehrens 2007), as in the case of the present study. Such analyses 
256 first produce a set of uncorrelated linear combinations of the original predictors, the PLS

257 components, calculated so as to maximize the covariance between the predictor and

258 response variables (Mevik and Wehrens 2007). Then, the PLS components are used to

259 derive the linear regression of the dependent variable (i.e. species or genetic diversity in

260 this case). To establish the optimal number of PLS components, we used a leave-one-out

261 cross-validation strategy (Mevik and Cederkvist 2004). Significance of the PLS model was

262 assessed by testing the significance of the Pearson correlation between the observed and

263 predicted values. To determine which habitat characteristics contributed most to the model,

264 we examined the loading weights and performed approximate t-tests based on jackknife

265 variance estimates of regression coefficients, calculated based on the optimal number of

266 PLS components.

267

268 Species and genetic diversity correlations

269 For each marker dataset, we computed Pearson correlations between plant species richness

270 and Nei's gene diversity index. One-tail tests with Bonferroni-Holm corrections were

271 applied to correct for multiple comparisons. In order to assess the overall trend in SGDCs,

272 we calculated Student's t-tests to determine whether the average correlation calculated from

273 the five species was greater than zero. To investigate whether the relationships between

274 species and genetic diversity were mediated by habitat isolation, stability, and size, we

275 performed partial Pearson correlations after controlling for significant effects of habitat

276 characteristics on species and genetic diversity. Significance of the residual correlations

277 was tested as described above for the raw SGDC coefficients. LMMs were performed to

278 test for differences in SGDCs between DS1 and DS2 (dataset as fixed effect), using species

279 as a random factor. 


\section{Results}

\section{Identification of the outlier loci}

282 Depending on the species, between 66 and 90 polymorphic AFLP loci were retained in our 283 study (Table S2). The BayeScan analysis identified between one and ten outlier loci per 284 species, representing between $1.2 \%$ and $15.1 \%$ of the AFLP loci, respectively, with an 285 average of $6.8 \%$ over all species (Table S2). Most of these outlier loci had high $F_{\mathrm{ST}}$ values, 286 but in $P$. clandestina and Austrelmis sp. two loci showed low $F_{\mathrm{ST}}$ values (Table S2).

\section{Species richness and genetic diversity}

289 Plant species richness ranged from 6 to 21 species per wetland (Table S3) and decreased 290 with latitude (Fig. S1). The two plant species showed low genetic diversity overall (range 291 of average Nei's gene diversity indices: $0.11-0.13$; Table S4), varying moderately to 292 substantially between wetlands (more than six-fold in C. gayana; Table S4). Genetic 293 diversity estimates of macroinvertebrates were higher (range of average Nei’s gene 294 diversity indices: 0.11-0.28; Table S4) but less variable (Table S4). The presence of outlier 295 loci significantly influenced Nei's gene diversity. These effects were detected in four of the 296 five species (Fig. 3), and as an overall trend across species (LMM, dataset effect: $L R=$ $29712.52, d f=1, P<0.001$ for DS1 vs DS2; $L R=24.77, d f=1, P<0.001$ for DS1 vs DS3).

298 The only species for which we failed to detect outlier loci effects on genetic diversity was

299 C. gayana, but only one outlier was identified for this species. Overall, GD estimates

300 decreased with the presence of outlier loci (Fig. 3, Fig. S1), and this trend was more 301 pronounced when only outliers with high $F_{\text {ST }}$ were included in the genetic datasets than 302 when both outliers with high and low $F_{\text {ST }}$ were included. 
304 Our analyses revealed positive correlations between species richness and genetic diversity

305 (Table S5). The average SGDCs of the five species was high (ranging from $0.51 \pm 0.07$ for

306 DS3 to $0.54 \pm 0.05$ for DS1, Table S5) and significantly positive overall ( $t$-tests: $t=17.4-$

$30725.5, d f=4, P<0.001$ in all cases). All SGDCs were positive and significant with respect

308 to each species $(P<0.05$ in all cases after correcting for multiple comparisons). They

309 ranged from 0.47 to 0.60 for DS1 (Table S5). The presence of outlier loci significantly

310 influenced the SGDCs (Fig. 4A, LMM, dataset effect: $L R=6.05, d f=2, P=0.05$ ), causing

311 a decrease in SGDCs overall (Fig 4A, Table S5). This trend was particularly marked in $P$.

312 clandestina (Fig. 4A), for which the SGDC estimate dropped by $13.5 \%$ when outlier loci

313 with high $F_{\text {ST }}$ were included (Fig. 4 A).

\section{Influence of habitat fragmentation on species richness, genetic diversity and SGDCs}

316 The PLS regressions explained $49 \%$ of the variation in wetland plant richness (Table 1).

317 Significant influence of wetland size and connectivity on genetic diversity was found for

318 the plants and the mayfly species (A. peruvianus), but no such effects were detected for the

319 long-lived aquatic species (H. fossamancinii and Austrelmis sp). Wetland isolation was an

320 important determinant of plant species richness and genetic diversity. We found a negative

321 influence of the average distance to neighboring patches (i.e. closeness centrality, Table 1)

322 and positive effects of the number of neighboring patches (i.e. node degree, Table 1) on

323 genetic and species diversity. A positive effect of the distance to the farthest wetland (i.e.

324 eccentricity) on species richness and genetic diversity was observed for the plants and the

325 mayfly species (Table 1). 
327 outlier loci (i.e. DS2 and DS3, Table 1). In fact, the opposite trend was observed in $P$.

328 clandestina and A. peruvianus, with habitat connectivity explaining a greater proportion of

329 the variance in GD for DS2/DS3 than for DS1 (Table 1).

330 Factoring out the effects of habitat fragmentation on species and genetic diversity

331 significantly influenced SGDC estimates (LMM, fragmentation habitat control effect: $L R=$

$3328.42, d f=1, P<0.01)$, decreasing average partial SDGCs relative to average original

333 values (Fig. 4B, Table S5). The decreases in partial SGDCs were more consistent and

334 pronounced with DS1 than with DS2 (Fig. 4B). In A. peruvianus, controlling for habitat

335 fragmentation had strikingly contrasting effects depending on whether or not the outlier loci

336 were included in the analysis (Fig. 4B). As in other species, the partial SGDC decreased

337 with DS1, but increased considerably with DS2, reaching a maximum of 0.7 (Table S5). To

338 confirm that this discrepancy was specifically linked to the three outlier loci detected, we

339 applied a bootstrap procedure to simulate the null distribution of the difference in partial

340 SGDCs between DS1 and DS2 when randomly eliminating three non-outlier loci from

341 DS2. None of the 1,000 bootstrap replications resulted in SGDC differences greater than or

342 equal to the one observed between DS1 and DS2.

\section{Discussion}

\section{Confounding effects of outlier loci in SGDC studies}

346

347

348 selection pressures; disregarding this fact can bias population genetic inferences (Luikart et

349 al. 2003, Landguth \& Balkenhol 2012). To avoid this problem, Luikart et al. (2003) 
advocated excluding $F_{\text {ST }}$ outlier loci from genetic analyses when the focus is on neutral

351 processes. In this study, we screened for AFLP outlier loci and investigated their impact on

352 SGDC estimates. We also examined how these loci influence the apparent contribution of

353 habitat characteristics linked to neutral processes (migration rates and rates of stochastic

354 loss of alleles/species) on SGDCs. Our results show that outlier loci led to a marked

355 decrease in GD, which in turn downplayed the strength of SGDCs.

356 Outlier loci have been suggested to be involved in adaptive processes (Hancock $e t$

357 al. 2011; Manel et al. 2012). Yet, alternative mechanisms, which may or may not be

358 indirectly associated with selective forces, have also been proposed to explain outlier loci,

359 such as correlations in co-ancestry between subpopulations in hierarchical structures, gene

360 surfing, background selection, and even endogenous genetic barriers (Bierne et al. 2011,

361 2013). Regardless of the actual mechanisms involved in the atypical of the actual

362 mechanisms involved in the atypical differentiation patterns of the outlier loci, those with

363 markedly high $F_{\mathrm{ST}}$ demonstrated the most pronounced effects in terms of reductions in GD.

364 This result concurs with previous empirical observations based on microsatellite data

365 (Garcia-Verdugo et al. 2015), and thus suggests that GD might often be underestimated

366 when such loci are included in population genetic analyses.

367 We found a significant downward effect of outlier loci on SGDCs, indicating that

368 SD correlates more strongly with GD of non-outlier loci than with GD of outlier loci. This

369 pattern is consistent with the expectation that SD is more closely related to neutral GD than

370 to non-neutral GD, due to the parallel influence of neutral processes on both diversity

371 levels. Investigating the effects of filtering out outlier loci from SGDC studies is therefore a

372 potentially useful approach to evidence the contribution of neutral processes. In addition, it

373 may contribute to revealing SGDCs, and explain some of the variation in species-genetic 
374 diversity relationships observed in the field. Indeed, the effects of outlier loci are likely to

375 depend on the strength of neutral mechanisms on both diversity components. In high

376 Andean wetlands, and highly fragmented ecosystems in general, neutral evolutionary

377 processes are likely key determinants of biodiversity. As a result, they are expected to

378 generate strong species-genetic diversity covariations (Vellend and Geber 2005; Whitlock

379 2014; Vellend et al. 2014), as were found here, with these SGDCs ranking among the

380 highest values reported to date (Vellend and Geber 2005; Whitlock 2014; Vellend et al.

381 2014). In such conditions, non-neutral processes, and thus outlier loci, should only have

382 minor confounding effects. The influence of outlier loci on SGDCs is likely to become

383 increasingly problematic as the relative importance of neutral processes in shaping diversity

384 patterns decreases. Extending the present framework to less fragmented ecosystems may

385 potentially uncover further SGDC patterns, thereby advancing current understanding of

386 species-genetic diversity relationships.

387 The presence of outlier loci also complicated the evaluation of the contribution to

388 SGDCs of habitat characteristics supposedly linked to neutral processes. Without such loci,

389 the partial SGDCs, calculated after filtering out the effects of habitat fragmentation on SD

390 and GD, decreased compared to the corresponding non-partial estimates. This indicates

391 concordant responses of SD and GD at non-outlier loci to these habitat characteristics.

392 Outlier loci somehow blurred these trends however, as reduction of partial SGDCs was less

393 consistent and less pronounced among species with outlier loci present. In the mayfly $A$.

394 peruvianus, the SGDC with outlier loci actually increased when habitat effects were

395 factored out (Fig. 4B). This indicates that contrasting effects of habitat characteristics on

396 SD and GD at outlier loci were partly masking the species-genetic diversity relationship in

397 this species. It further shows that GD at outlier loci and neutral GD can correlate differently 
with habitat features, thus confounding the apparent importance of neutral mechanisms on

399 GD. In fact, for both $P$. clandestina and A. peruvianus, the habitat features showed stronger 400 correlations with GD at outlier than at non-outlier loci (Table 1). Habitat stability, size, and

401 connectivity, being intrinsic determinants of population demography, are expected to

402 influence all neutral loci equally (Luikart et al. 2003). Their stronger association with

403 outlier loci in two species thus suggests that they can also be indirectly associated with

404 selective processes and local adaptation. In our case, high altitude wetland densities decline

405 in parallel with latitude, and both wetland size and connectivity show spatial structure along

406 this gradient (Fig. S2, Supplementary Material). In such circumstances, the standard

407 approach to disentangling the influence of neutral processes on SGDCs - i.e., factoring out

408 the effects of habitat features- may be ineffective, since it can simultaneously omit the

409 effects of selective environmental factors. Our results thus illustrate the difficulties in

410 inferring evolutionary processes from partial SGDCs when habitat density is paired with

411 environmental gradient, a situation likely to be common in ecosystems subject to climatic

412 and/or topographic influence. To overcome pitfalls in interpreting the contribution of

413 disturbance regime, habitat size and/or connectivity to SGDCs, it would be advisable to

414 analyze the spatial distribution of these variables and evaluate their correlation with other

415 environmental factors as potential actors of selection.

417 Species-genetic diversity correlations in high altitude wetlands: patterns and processes

418 We found strong spatial associations between species richness and AFLP genetic diversity

419 estimates in high altitude wetlands. Consistent with simulation models suggesting stronger

420 species-genetic diversity relationships when GD is measured in more common species

421 (Vellend 2005), highest SGDC was obtained for C. gayana, the most abundant plant 
422 species of high altitude wetlands of Chile's Norte Chico. Overall, our results add to

423 previous meta-analysis evidence that SGDCs are widespread in discrete habitats

424 functioning as islands (Vellend and Geber 2005; Whitlock 2014; Vellend et al. 2014).

425 Furthermore, our results show that such correlations are not limited to component species

426 of the community. For instance, we detected strong SGDCs between plant species richness

427 and GD of the benthic macro-invertebrate taxa, despite the fact that the latter possess very

428 different life-history traits, particularly regarding their dispersal modes.

429 SGDCs in patchy ecosystems are thought to result from parallel influences of

430 neutral forces governing variation at the genetic and species diversity levels (Vellend and

431 Geber 2005; Whitlock 2014; Vellend et al. 2014). Here, the contribution of such

432 mechanisms is substantiated both by the lowering effect of outlier loci on SGDCs, as well

433 as the apparent common responses of SD and GD at non-outlier loci to habitat features

434 (size, connectivity and stability). The persistence of positive, and overall significant, partial

435 SGDCs after removing all confounding effects (outlier loci and habitat features) suggests,

436 however, that other causal mechanisms might also be involved. These can include both

437 parallel responses to processes unrelated to habitat features, and direct relationships

438 between SD and GD. While our results indicate that potentially selected loci tend to

439 decrease SGDCs overall, they do not rule out the possibility that some selective

440 mechanisms may contribute to species-genetic diversity relationships. For instance, a strong

441 selective agent, e.g. a voracious herbivore or an aggressive exotic plant, acting with

442 increasing intensity upon plant communities located along a spatial gradient might cause a

443 gradual reduction in SD (due to differences in species' abilities to cope with increased

444 predation/competition) together with a reduction of GD in the "surviving" species, where

445 only some tolerant genotypes could remain; this would generate a positive correlation 
446 between SD and GD. In our study system, plant SD may affect soil and water

447 characteristics and, as such, may influence local GD by determining the abundance of plant 448 and macroinvertebrate populations. Alternatively, genetic variation within foundation

449 species such as $P$. clandestina, which hosts other plant species, can affect the composition 450 and diversity of associated communities (Whitham et al. 2006). Nevertheless, this

451 conjecture would imply a significant correlation between GD at non-outlier loci and GD of

452 specific genes encoding ecologically relevant traits influencing plant assemblages.

453 Further investigation is required to fully unravel the mechanisms driving SGDCs in

454 high Andean wetlands. Future studies will benefit from next-generation sequencing applied 455 to genome scans, which will very soon allow for more reliable estimates of genes under 456 selection (Manel et al. 2016), essential to distinguishing neutral from adaptive markers.

457 Ideally, field studies could complement these efforts by identifying strong interactions 458 between species in the community, putative selective pressures, and patterns of selection 459 and adaptive evolution.

\section{Acknowledgements}

462 We thank F. Squeo, G. Arancio, D. Rodriguez, R. Inzulza Ayala, L. Cifuentes, E. Alvarez, 463 C. Urqueta, R. Hereme and A. Troncoso for their help with field work and/or for their 464 technical assistance, and Craig Weideman for revising the English of the manuscript. Our 465 thanks go to P. Jarne and F. Laroche for insightful discussion during the preparation of this 466 manuscript. We also thank four anonymous reviewers for their comments on previous 467 versions of the manuscript. The authors are grateful to La Junta de Vigilancia del Elqui, la 468 Comunidad Agrícola los Huascoaltinos, La Sociedad de Parceleros de Coiron, La Sociedad 
de Parceleros de San Agustín, La Sociedad de Parceleros Hacienda Illapel, la Hacienda

470 Tulahuén Oriente, la Hacienda El Maiten de Pedregal and la Hacienda El Bosque for giving

471 us access to private wetlands. This research was funded by a grant from the Chilean

472 National Research Agency FONDECYT \#1110514 and the exchange research grant ECOS-

473 CONICYT C12B02.

\section{References}

476 Antonovics J (1992) Toward community genetics. In: Plant resistance to herbivores and 477 pathogens: ecology, evolution, genetics (eds. Fritz RS, Simms E), pp. 426-449. The $478 \quad$ University of Chicago Press, Chicago.

479 Arroyo MTK, Marquet P, Marticorena C et al. (2004) Chilean winter rainfall-Valdivian 480 forests. In: Hotspots revised: Earth's biologically wealthiest and most threatened 481 ecosystems. (eds. Mittermeier RA, Gil PR, Hoffmann M et al.), pp. 99-103. CEMEX, $482 \quad$ México D.F.

483 Barton NH (2000) Genetic hitchhiking. Philosophical Transactions of the Royal Society of 484 London Series B-Biological Sciences, 355, 1553-1562.

485 Bertin A, Alvarez E, Gouin N et al. (2015) Effects of wind-driven spatial structure and environmental heterogeneity on high-altitude wetland macroinvertebrate assemblages

490 Bierne N, Roze D, Welch JJ (2013). Pervasive selection or is it ...? Why are Fst outliers sometimes so frequent? Molecular Ecology, 22, 2061-2064. 
Bonin A, Ehrich D, Manel S (2007) Statistical analysis of amplified fragment length polymorphism data: a toolbox for molecular ecologists and evolutionists 6. Molecular Ecology, 16, 3737-3758.

Duchesne P, Bernatchez L (2002) AFLPOP: a computer program for simulated and real population allocation, based on AFLP data. Molecular Ecology Notes, 2, 380-383.

Eckert C, Samis K, Lougheed S (2008) Genetic variation across species' geographical ranges: the central-marginal hypothesis and beyond. Molecular Ecology, 17, 11701188.

Ehrich D (2006) AFLPDAT: a collection of R functions for convenient handling of AFLP data. Molecular Ecology Notes, 6, 603-604.

Foll M, Gaggiotti O (2008) A Genome-Scan Method to Identify Selected Loci Appropriate for Both Dominant and Codominant Markers: A Bayesian Perspective. Genetics, 180, 977-993.

Foltete JC, Clauzel C, Vuidel G (2012) A software tool dedicated to the modelling of landscape networks. Environmental Modelling \& Software, 38, 316-327.

Frankham R (1996) Relationship of genetic variation to population size in wildlife. Conservation Biology, 10, 1500-1508.

Frankham R (1997) Do island populations have less genetic variation than mainland populations? Heredity, 78, 311-327.

García-Verdugo C, Sajeva M, La Mantia T et al. (2015) Do island plant populations really have lower genetic variation than mainland populations? Effects of selection and distribution range on genetic diversity estimates. Molecular Ecology, 24, 726-741.

Hancock AM, Brachi B, Faure N et al. (2011) Adaptation to Climate Across the Arabidopsis thaliana Genome. Science, 334, 83-86. 
He T, Lamont BB, Krauss SL, Enright NJ, Miller BP (2008) Covariation between intraspecific genetic diversity and species diversity within a plant functional group. Journal of Ecology, 96, 956-961.

Holderegger R, Kamm U, Gugerli F (2006) Adaptive vs. neutral genetic diversity: implications for landscape genetics. Landscape Ecology, 21, 797-807.

Hu FS, Hampe A, Petit RJ (2009) Paleoecology meets genetics: deciphering past vegetational dynamics. Frontiers in Ecology and the Environment, 7, 371-379.

Hubbell, S.P. (2001) The Unified Neutral Theory of Biodiversity and Biogeography, Princeton University Press.

Johnson MTJ, Stinchcombe JR (2007) An emerging synthesis between community ecology and evolutionary biology. Trends in Ecology \& Evolution, 22, 250-257.

Kahilainen A, Puurtinen M, Kotiaho JS (2014) Conservation implications of speciesgenetic diversity correlations. Global Ecology Conservation, 2, 315-323.

Kirk H, Freeland JR (2011) Applications and Implications of Neutral versus Non-neutral Markers in Molecular Ecology. International Journal of Molecular Sciences, 12 , 3966-3988.

Lamy T, Jarne P, Laroche F et al. (2013) Variation in habitat connectivity generates positive correlations between species and genetic diversity in a metacommunity. Molecular Ecology, 22, 4445-4456.

Lankau RA, Strauss SY (2007) Mutual feedbacks maintain both genetic and species diversity in a plant community. Science, 317, 1561-1563.

Laroche F, Jarne P, Lamy T, David P, Massol F (2015) A Neutral Theory for Interpreting Correlations between Species and Genetic Diversity in Communities. American Naturalist, 185, 59-69. 
Lavergne S, Mouquet N, Thuiller W, Ronce O (2010) Biodiversity and Climate Change: Integrating Evolutionary and Ecological Responses of Species and Communities. Annual Review of Ecology, Evolution, and Systematics, Vol 41, 41, 321-350.

Luikart G, England PR, Tallmon D, Jordan S, Taberlet P (2003) The power and promise of population genomics: From genotyping to genome typing 13. Nature Reviews Genetics, 4, 981-994.

MacArthur R.H., Wilson E.O. (1967) The theory of island biogeography. Princeton University Press, New Jersey.

Manel S, Perrier C, Pratlong M et al. (2016) Genomic resources and their influence on the detection of the signal of positive selection in genome scans. Molecular Ecology, 25, 170-184.

Manel S, Gugerli F, Thuiller W et al. (2012) Broad-scale adaptive genetic variation in alpine plants is driven by temperature and precipitation. Molecular Ecology, 21,

554 Meudt HM, Clarke AC (2007) Almost forgotten or latest practice? AFLP applications, analyses and advances. Trends in Plant Science, 12, 106-117.

Mevik BH, Cederkvist HR (2004) Mean squared error of prediction (MSEP) estimates for principal component regression (PCR) and partial least squares regression (PLSR). Journal of Chemometrics, 18, 422-429.

Mevik BH, Wehrens R (2007) The pls package: Principal component and partial least squares regression in R. Journal of Statistical Software, 18, 1-23.

561 Mouquet N, Devictor V, Meynard CN et al. (2012) Ecophylogenetics: advances and 562 perspectives. Biological Reviews, 87, 769-785. 
563 Nei M (1987) Molecular Evolutionary Genetics. Columbia University Press, New York, N.

$564 \quad \mathrm{Y}$.

565 Odat N, Jetschke G, Hellwig FH (2004) Genetic diversity of Ranunculus acris L.

566 (Ranunculaceae) populations in relation to species diversity and habitat type in 567 grassland communities. Molecular Ecology, 13, 1251-1257.

568 Oleksyk TK, Smith MW, O'Brien SJ (2010) Genome-wide scans for footprints of natural 569 selection. Philosophical Transactions of the Royal Society B-Biological Sciences, $570 \quad 365,185-205$.

571 Papadopoulou A, Anastasiou I, Spagopoulou F et al. (2011) Testing the Species-Genetic 572 Diversity Correlation in the Aegean Archipelago: Toward a Haplotype-Based 573 Macroecology? American Naturalist, 178, 241-255.

574 R Core Team. A language and environment for statistical computing. 2015. Vienna, $575 \quad$ Austria, R Foundation for Statistical Computing.

576 Ricklefs RE, Lovette IJ (1999) The roles of island area per se and habitat diversity in the 577 species-area relationships of four Lesser Antillean faunal groups. Journal of Animal $578 \quad$ Ecology, 68, 1142-1160.

579 Rosenzweig (1995) Species diversity in space and time, Cambridge University Press $580 \quad$ ednCambridge, UK.

581 Rossi J (2011) Rich: an R package to analyse species richness. Diversity, 3, 112-120.

582 Rouse JW, Haas RH, Schell J, Deering D (1973) Monitoring vegetation systems in the 583 Great Plains with ERTS. In: Third ERTS Symposium (ed. NASA SP-351 I), pp. 309317. 
Silvertown J, Biss PM, Freeland J (2009) Community genetics: resource addition has opposing effects on genetic and species diversity in a 150-year experiment. Ecology Letters, 12, 165-170.

Squeo FA, Warner BG, Aravena R, Espinoza D (2006) Bofedales: high altitude peatlands of the central Andes. Revista Chilena de Historia Natural, 79, 245-255.

Storz JF, Payseur BA, Nachman MW (2004) Genome scans of DNA variability in humans reveal evidence for selective sweeps outside of Africa. Molecular Biology and Evolution, 21, 1800-1811.

Strauss SY, Sahli H, Conner JK (2005) Toward a more trait-centered approach to diffuse (co)evolution. New Phytologist, 165, 81-89.

Struebig MJ, Kingston T, Petit EJ et al. (2011) Parallel declines in species and genetic diversity in tropical forest fragments. Ecology Letters, 14, 582-590.

Taberlet P, Zimmermann NE, Englisch T et al. (2012) Genetic diversity in widespread species is not congruent with species richness in alpine plant communities. Ecology Letters, 15, 1439-1448.

Vellend M (2004) Parallel effects of land-use history on species diversity and genetic diversity of forest herbs. Ecology, 85, 3043-3055.

Vellend M (2005) Species diversity and genetic diversity: Parallel processes and correlated patterns. American Naturalist, 166, 199-215.

Vellend M, Geber MA (2005) Connections between species diversity and genetic diversity. Ecology Letters, 8, 767-781.

Vellend M, Lajoie G, Bourret A et al. (2014) Drawing ecological inferences from coincident patterns of population- and community-level biodiversity. Molecular Ecology, 23, 2890-2901. 
609 Whitham TG, Bailey JK, Schweitzer JA et al. (2006) A framework for community and 610 ecosystem genetics: from genes to ecosystems. Nature Reviews Genetics, 7, 510-523.

611 Whitlock R (2014) Relationships between adaptive and neutral genetic diversity and 612 ecological structure and functioning: a meta-analysis. Journal of Ecology, 102, 857$613 \quad 872$.

$614 \mathrm{Xu} \mathrm{W}$, Liu L, He T et al. (2016) Soil properties drive a negative correlation between 615 species diversity and genetic diversity in a tropical seasonal rainforest. Scientific 616 Reports, 6.

618 Data accessibility

619 Raw files of the AFLP genotypes (i.e. band presence and absence) for each of the five 620 species and R scripts will be made available from the Dryad Digital Repository upon 621 acceptance of the manuscript.

623 Author contributions

624 ABertin, NG, ABaumel, EG and SM conceived and designed the study. ABertin, NG, JS 625 and RO performed the experiments. ABertin, NG and ABaumel analyzed the data. ABertin, $626 \mathrm{NG}, \mathrm{ABaumel}$ and SM wrote the manuscript. All the authors contributed substantially to 627 revisions.

628 


\section{Tables}

630 Table 1. Effects of habitat characteristics on genetic diversity and taxa diversity estimated using partial least-square regressions

631 (PLSR). The direction of the effect (positive/negative) of each habitat characteristic on the diversity parameters is reported in

632 parentheses. DS1 refers to the AFLP dataset excluding outlier loci, DS2 to the dataset including both non-outlier and outlier loci

633 (potentially under diversifying or balancing selection), and DS3 to the dataset including non-outlier and outlier loci with high $F_{\text {ST }}$

634 (potentially under diversifying selection).

\begin{tabular}{|c|c|c|c|c|c|}
\hline & & & Optimal & & Explained \\
\hline & AFLP & Number of & number of & & variance \\
\hline & dataset & sites & PLSR axes & Most contributing variables & $\left(R^{2}\right)$ \\
\hline Species diversity & & 21 & 3 & Closeness centrality $(<0)$, Eccentricity $(>0)$, Node degree $(>0)$ & $0.49 * * *$ \\
\hline \multicolumn{6}{|l|}{ Genetic Diversity } \\
\hline \multirow[t]{2}{*}{ C. gayana } & DS1 & 21 & 3 & Node degree $(>0)$, Closeness centrality $(<0)$, Eccentricity $(>0)$ & $0.51 * * *$ \\
\hline & DS2 / DS3 & 21 & 3 & Node degree $(>0)$, Closeness centrality $(<0)$, Eccentricity $(>0)$ & $0.50 * * *$ \\
\hline P. clandestina & DS1 & 16 & 1 & Node degree $(>0)$, Capacity $(>0)$ & $0.19^{\dagger}$ \\
\hline
\end{tabular}




\begin{tabular}{|c|c|c|c|c|c|}
\hline & DS2 & 16 & 1 & Closeness centrality $(<0)$, Eccentricity $(>0)$, Node degree $(>0)$, & $0.35^{*}$ \\
\hline & & & & Capacity $(>0)$ & \\
\hline & DS3 & 16 & 2 & Node degree $(>0)$, Capacity $(>0)$ & $0.31^{\dagger}$ \\
\hline \multirow[t]{2}{*}{ H. fossamancinii } & DS1 & 14 & 0 & - & - \\
\hline & DS2 / DS3 & 14 & 0 & - & - \\
\hline \multirow[t]{2}{*}{ A. peruvianus } & DS1 & 14 & 0 & - & - \\
\hline & DS2 / DS3 & 14 & 1 & Closeness centrality $(<0)$ & $0.43 *$ \\
\hline \multirow[t]{3}{*}{ Austrelmis sp } & DS1 & 15 & 0 & - & - \\
\hline & DS2 & 15 & 0 & - & - \\
\hline & DS3 & 15 & 0 & - & - \\
\hline
\end{tabular}

$635: P<0.1, *: P<0.05$ and $* * *: P<0.001$ 


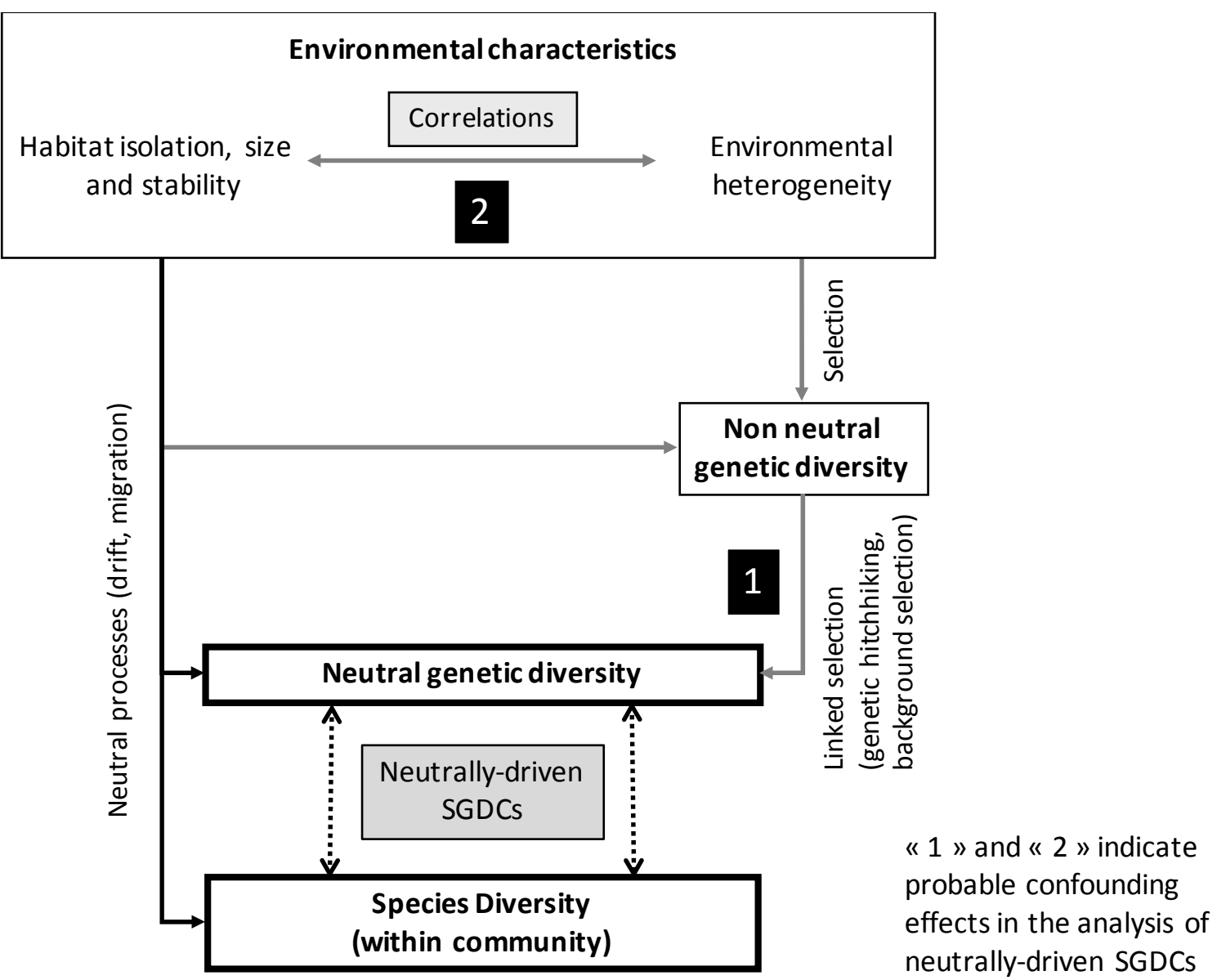

Figure 1. Relationship between species diversity within a community and neutral genetic diversity within a species. Species-genetic diversity correlations (SGDCs) with neutral genetic diversity are assumed to result from parallel responses to neutral processes (black arrows), which relative importance is expected to be determined by habitat features such as disturbance regime, habitat size and/or connectivity. Yet, non-neutral processes can have confounding effects (grey arrows) in the investigation of neutrally-driven SGDCs. They can confound the apparent level of SGDCs driven by neutral processes (confounding effect 1) influencing neutral genetic diversity through linked selection. The contribution of habitat characteristics regulating rates of stochastic immigration and extinction to SGDCs may also be confounded by linked selection if adaptive genetic markers are selected by environmental conditions covarying with those habitat characteristics (confounding effect 2). 


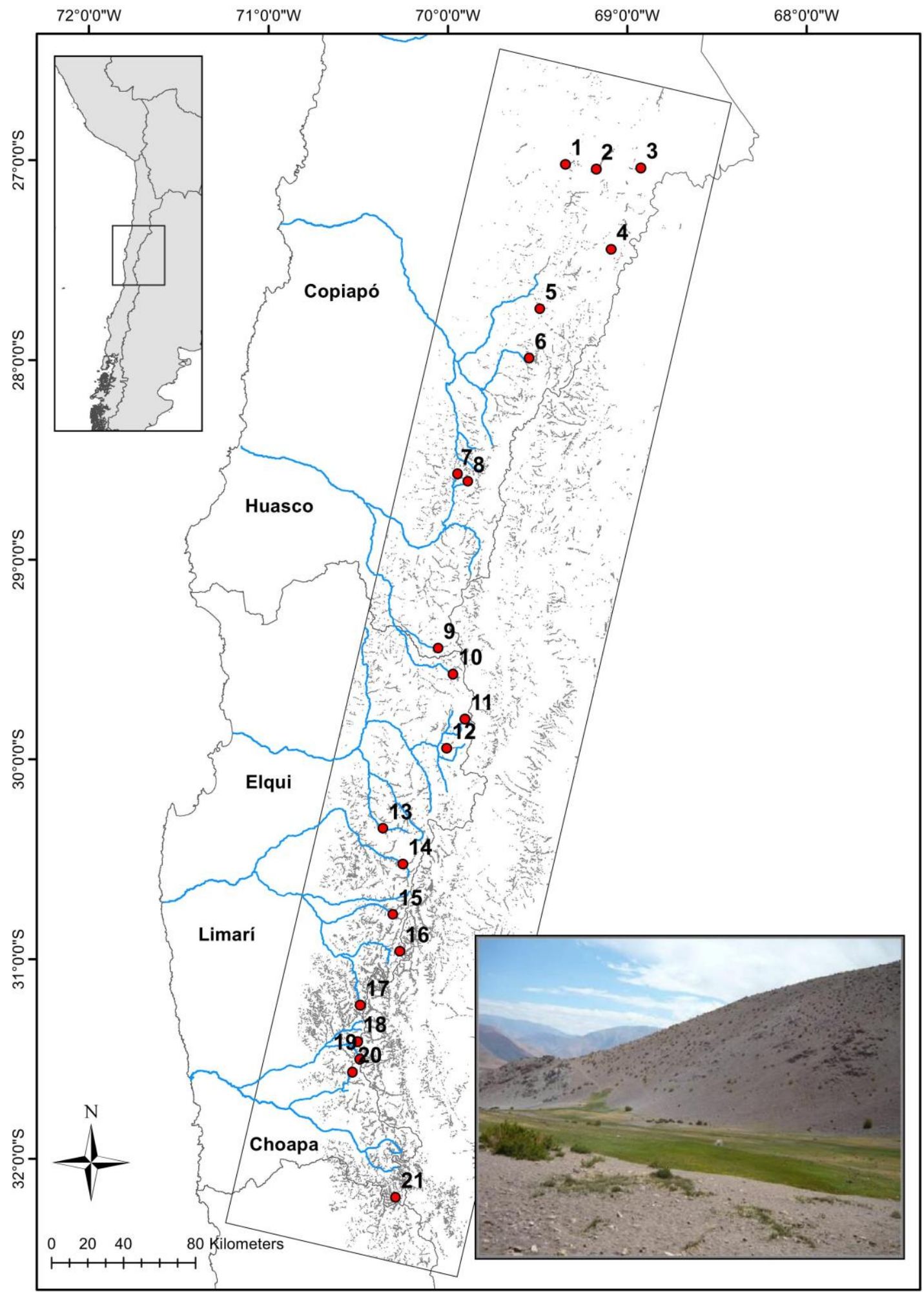

651 Figure 2. Picture of a high altitude wetland and geographical distribution of the sampling 652 sites along Chile's Norte Chico. The rectangle depicts the remote-sensing area used for 653 identification and mapping of high Andean wetlands (shown in grey). 


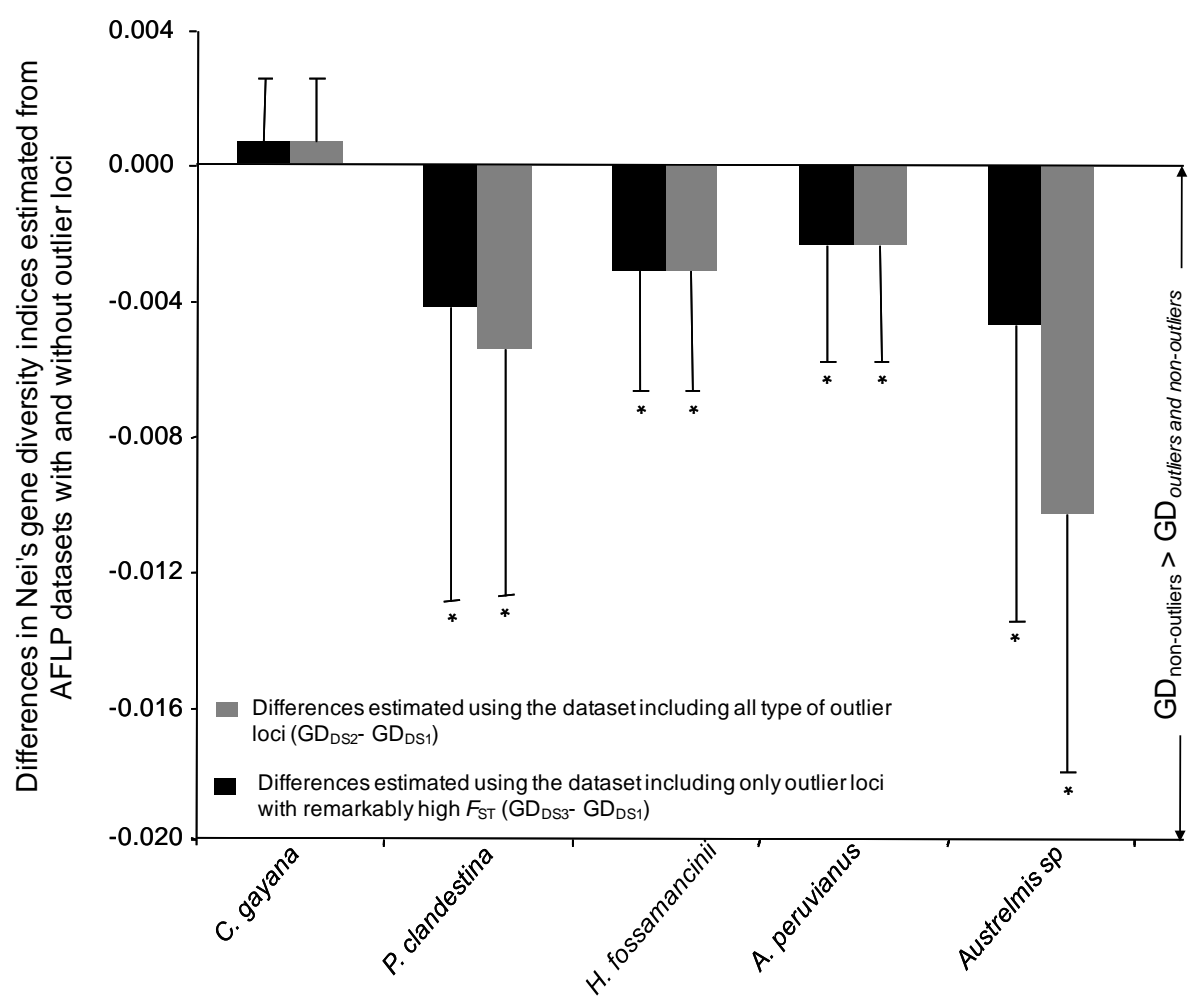

Figure 3. Average population difference + or - SD between Nei's gene diversity indices estimated from the AFLP datasets including outlier loci (DS2 and DS3) and the dataset free of outliers (DS1). Asterisks indicate significant differences $(P<0.05)$ in average population genetic diversity estimated with and without outlier loci for each comparison in each

659 species. 

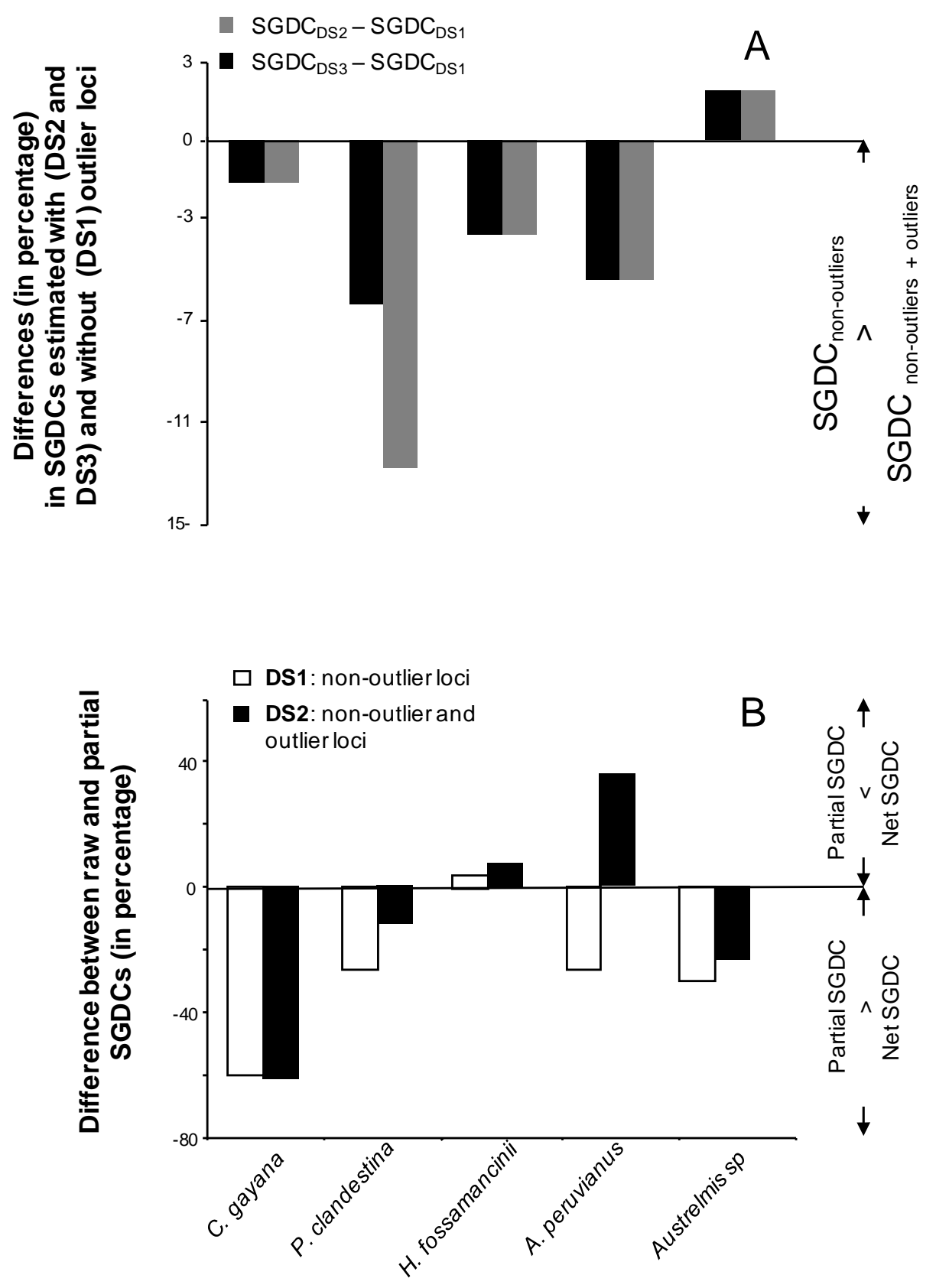

Figure 4. Differences between species genetic diversity correlations (SGDCs) estimated under various scenarios. A) Differences between SGDCs estimated without (SGDC DS1 $_{1}$ ) and with outlier loci (SGDC $\mathrm{DS}_{\mathrm{D} 2}$ and $\mathrm{SGDC}_{\mathrm{DS} 3}$ ). $\mathrm{SGDC}_{\mathrm{DS} 2}-\mathrm{SGDC}_{\mathrm{DS} 1}$ refers to the difference between SGDCs calculated with the full AFLP dataset including both non-outlier and outlier loci (DS2) and the dataset excluding all the outlier loci (DS1), and $\mathrm{SGDC}_{\mathrm{DS} 3}-$ $\mathrm{SGDC}_{\mathrm{DS} 1}$ to the difference between SGDCs calculated with the dataset including nonoutlier and outlier loci with high $F_{\text {ST }}$ only (DS3) and the dataset excluding all the outlier loci (DS1). B) Difference between raw SGDCs and partial SGDCs, calculated after accounting for habitat characteristics, for each focal species and for datasets without (DS1) and with all the outlier loci (DS2). 